Article

\title{
Injection Molding Process Control of Servo-Hydraulic System
}

\author{
Chun-Ying Lin ${ }^{1}$, Fang-Cheng Shen ${ }^{1,+}{ }^{,}$Kuo-Tsai $\mathrm{Wu}^{1}$ and Huei-Huang Lee ${ }^{2}$ \\ and Sheng-Jye Hwang ${ }^{1, *}$ \\ 1 Department of Mechanical Engineering, National Cheng Kung University, Tainan 70101, Taiwan; \\ linleo1024@gmail.com (C.-Y.L.); iamagoodguy10028@gmail.com (F.-C.S.); adiktwu@gmail.com (K.-T.W.) \\ 2 Department of Engineering Science, National Cheng Kung University, Tainan 70101, Taiwan; \\ hhlee@mail.ncku.edu.tw \\ * Correspondence: jimppl@mail.ncku.edu.tw \\ † Current address: No. 1, University Road, Tainan 70101, Taiwan.
}

Received: 31 October 2019; Accepted: 15 December 2019; Published: 20 December 2019

\begin{abstract}
The present study constructs a servo-hydraulic system to simulate the filling and packing processes of an injection molding machine. Experiments are performed to evaluate the velocity and position control of the system in the filling stage and the pressure control in the packing stage. The results demonstrate that the proposed system meets the required performance standards when operated with the proportional-integral-derivative (PID) controller under a sampling frequency of $1000 \mathrm{~Hz}$
\end{abstract}

Keywords: injection molding; position control; pressure control; V-P transition; servo-hydraulic system; PID controller

\section{Introduction}

Servo-hydraulic systems have many advantages for injection molding machines, including high precision, low noise, a low operating temperature, and good repeatability [1-3]. Furthermore, compared with traditional hydraulic systems, servo-hydraulic systems have the potential to achieve energy savings of up to $70 \%$, or more [4,5]. However, to achieve this energy-saving potential, the servo-hydraulic system must first be carefully designed and optimized.

Of all the parameters involved in the injection molding process, the injection velocity and packing pressure are two of the most important ones in determining the quality of the molded part. The injection velocity refers to the speed at which the screw moves in the forward direction during the cavity filling stage, while the packing pressure refers to the pressure applied to the molten resin in the cavity as it cools and solidifies to form the final component. However, even when the injection velocity and packing pressure are properly controlled, the accuracy with which the injection cycle transits from the constant-velocity $(\mathrm{V})$ filling the stage to the constant-pressure $(\mathrm{P})$ packing stage plays a critical role in ensuring a correct and consistent quality of the molded component [6,7]. Many approaches have been proposed for controlling the V-P switchover point, including pressure switch, time switch and position switch. In the position control method, V-P switchover is initiated automatically as soon as the screw displacement reaches a certain predetermined value [8-10]. Among all the methods available, the position control method is one of the most commonly used since, the same amount of resin is injected into the mold in each shot, and hence the consistency of the molded part quality is guaranteed [11].

Previous studies have reported that moving the screw backward through a short distance when reaching the V-P transition point is beneficial in decreasing the pressure at the screw front and hence 
in preventing overflow or part warpage [12]. Kamiguchi et al. [13] had illustrated how the pull-back motion work, yet the discussion including the distance and performances of pull-back motion have been omitted. Particularly, pulling the screw back too far runs the risk of trapping air bubbles in the mold, while moving the screw back too little risks not reaching the pressure reduction function. Hence, the pull-back distance must be carefully controlled to ensure that the molded components satisfy the required quality standard.

The present study designs and constructs a servo-hydraulic system to simulate the filling and packing stages of an injection molding machine. A proportional-integral-derivative (PID) controller is additionally designed to control the V-P switchover point using the position control method. Experiments were performed to investigate the performance of the proposed system in terms of its speed control (filling process), pressure control (packing process) and V-P switchover point control (V-P transition). In general, the results confirm the feasibility of the proposed servo-hydraulic system for injection molding applications given an appropriate setting of the sampling frequency $(1000 \mathrm{~Hz})$.

\section{Materials and Methods}

\subsection{Experimental System}

Figures 1 and 2 presents a schematic illustration of the experimental system constructed in the present study. The micro control unit, as known as MCU, is produced by ICP DAS company with $1 \mathrm{kHz}$ sampling rate, seven channels to install analog I/O, digital I/O modules. The driver which had a 20-bits resolution encoder is produced by the DELTA company. The servo motor is a permanent-magnetic motor produced by DELTA company, and its power is $5.5 \mathrm{~kW}$ with a maximum motor speed of $3000 \mathrm{rpm}$. The power source in the experiment system was a piston pump from the YEOSHE company with the highest pump speed is $2750 \mathrm{rpm}$. Additionally, the continuous working pressure and displacement of the pump were respectively $350 \mathrm{bar}$ and $16 \mathrm{cc} / \mathrm{rev}$. More details of main components' specifications are listed in Table 1.

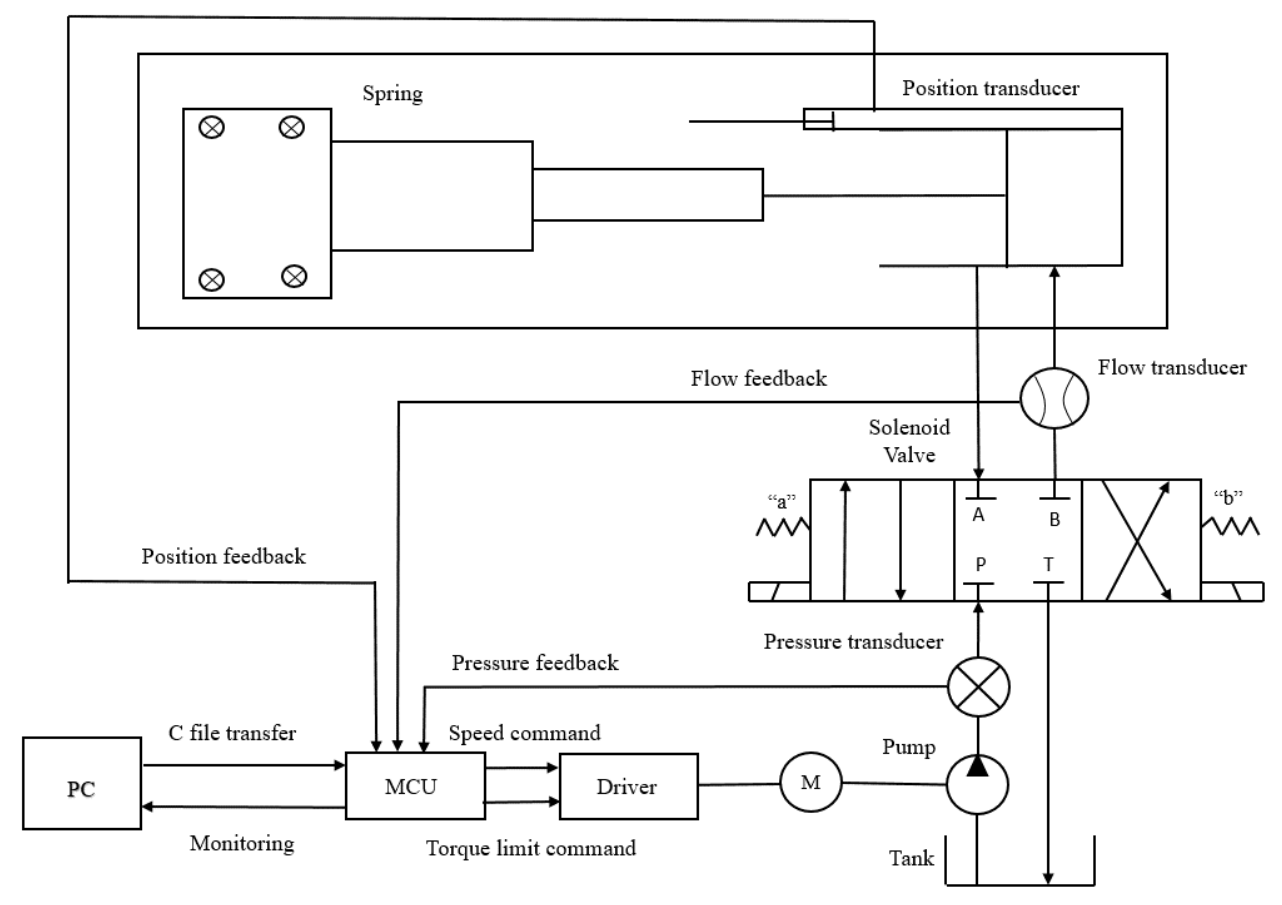

Figure 1. Experimental system.

The servo proportional valve is a key component in the proposed system, which changes the opening size according to the input voltage. The valve is produced by Parker, which has a maximum 
flow rate of $90 \mathrm{~L} / \mathrm{min}$, receives a diagnostic signal from $+10 \mathrm{~V}$ to $-10 \mathrm{~V}$ and the frequency response of $350 \mathrm{~Hz}$. Servo valves operate with very high accuracy, very high repeatability and very high-frequency response, even so, the servo proportional valve has acceptable performance and lower cost for industrial usage. A nitrogen gas spring was installed in the experiment. When the screw moved forward, it encountered a resistance force, which was the role that the spring plays. This loading experiment system was applied to simulate the situation between the injection and the packing stage. This nitrogen gas spring is produced by NITROGAS company. The maximum compression stroke is $125 \mathrm{~mm}$ and its maximum working pressure is 150 bar.

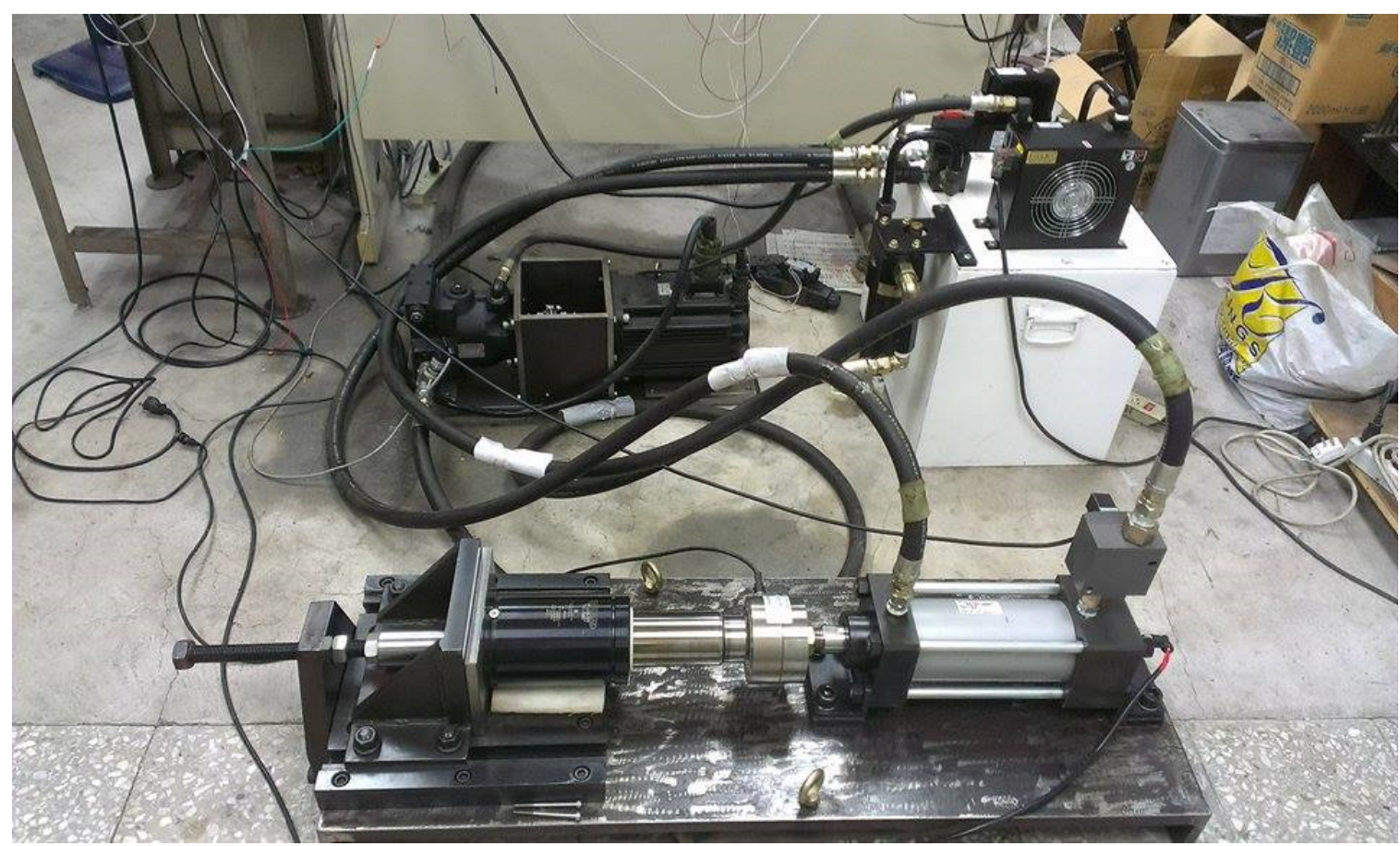

Figure 2. The outfit of the experimental system.

Table 1. Main components' specifications of the experiement system.

\begin{tabular}{ll}
\hline Components & Specifications \\
\hline Hydraulic cylinder & Diameter: $100 \mathrm{~mm}$ \\
& Max. of stroke: $170 \mathrm{~mm}$ \\
& Max. working pressure: $140 \mathrm{bar}$ \\
Servo proportional valve & Max. flow rate: $90 \mathrm{~L} / \mathrm{min}$ \\
& Max. working pressure: $350 \mathrm{bar}$ \\
& Step response: $3.5 \mathrm{~ms}$ \\
Micro control unit & OS: Linux kernel 2.6 .33 \\
Position transducer & Resolution (Analog): $12 \mathrm{bit}$ \\
\hline
\end{tabular}

\subsection{Control Strategy}

It is well known that the PID controller has been widely accepted for controlling the industrial system.

Figure 3 shows the control strategy developed in the present study to control the servo-hydraulic system in the constant velocity and constant pressure modes. In the filling (constant velocity) stage, the controller takes the feedback signal provided by a flow meter as the input and adjusts the servo-motor speed in accordance with a prescribed control law such that the velocity remains at the required value. Once the hydraulic stage reaches the target switchover position, the controller reduces the voltage output in order to reduce the motor speed and enters the pressure control stage. 
Subsequently, the controller maintains a constant pressure by advancing the hydraulic stage slowly in accordance with the signals received from a pressure transducer and torque sensor.

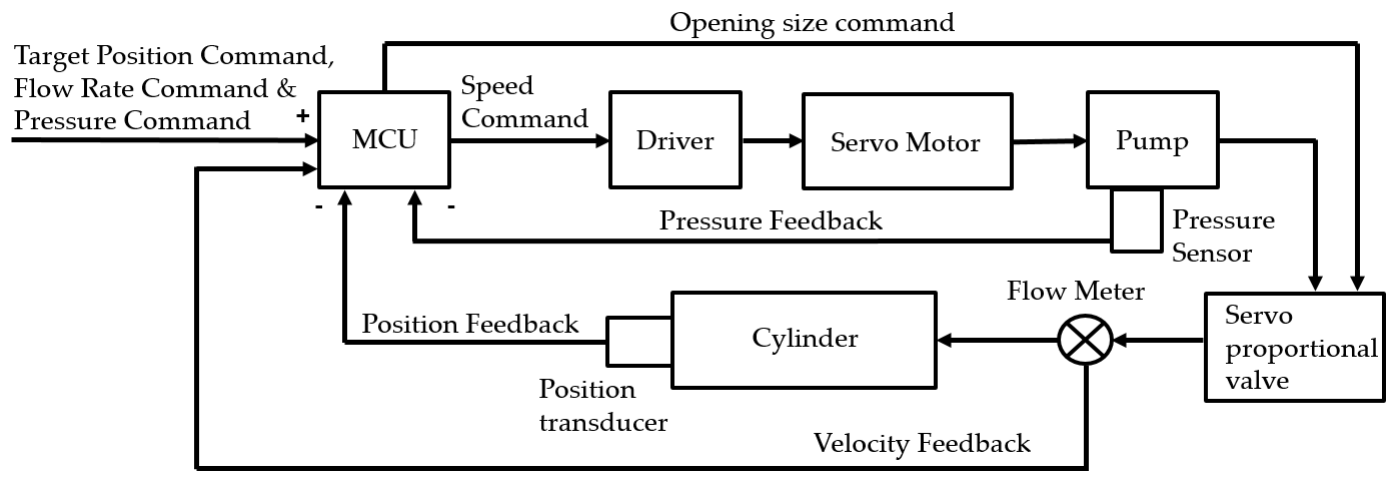

Figure 3. Flowchart of proposed control strategy.

Figure 4 indicates how the servo proportional valve acts in the experiment. The servo proportional valve is set to fully open in the initial from node P to B (see Figure 1) by applying the voltage $-10 \mathrm{~V}$. Once the position of the hydraulic cylinder reached to the first target position, set the opening size to $0.5 \%$ (applied the voltage of $-0.05 \mathrm{~V}$ ). Next, switch the node P to A (applied the voltage of $0.25 \mathrm{~V}$ to the valve) in order to perform the pull-back motion.

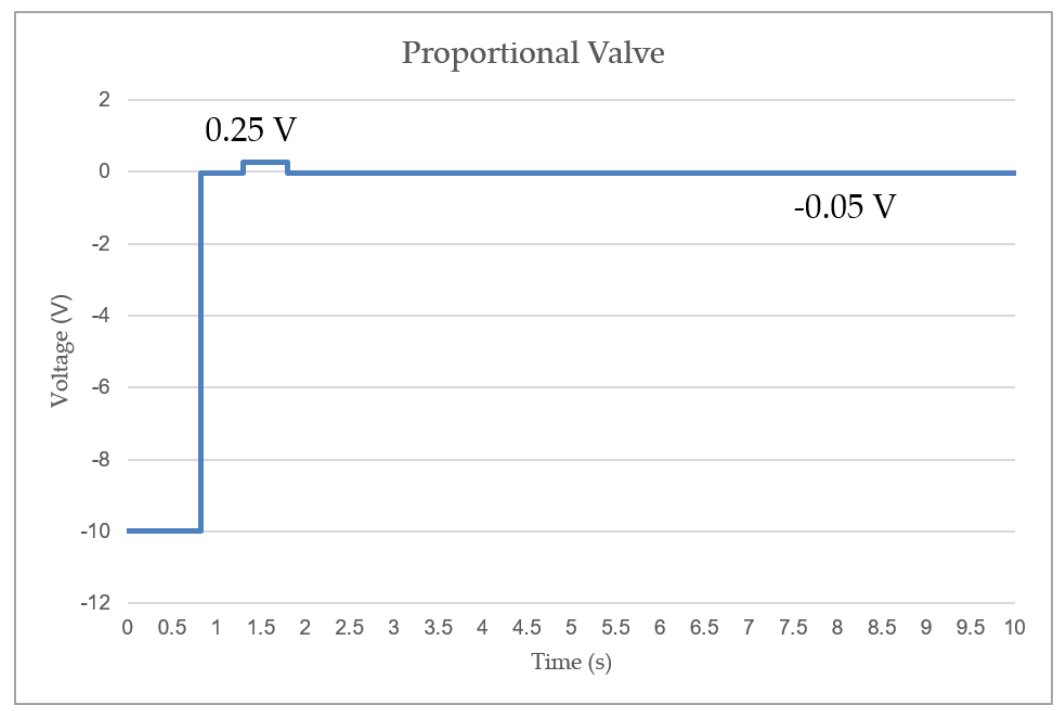

Figure 4. Voltage input to the servo proportional valve during the control sequence.

\subsection{Experimental Performance Indicators and Targets}

The maximum overshoot was defined as the difference between the maximum recorded pressure value and the target pressure value. Meanwhile, the response time was defined as the time taken for the cylinder to move backward to the second target position having first reached the V-P switchover position. Finally, the standard deviation (RMSD) of the pressure was defined as the magnitude of the pressure variation between the system pressure and the target pressure under steady-state conditions.

The target values are based on industrial demands. In performing the experiments, the target value for the maximum overshoot of the pressure at the hydraulic cylinder was set as less than 5 bar, the overshoot of the cylinder position as less than $1 \mathrm{~mm}$, the response time as less than $3 \mathrm{~s}$, the RMSD of the cylinder position as less than $0.5 \mathrm{~mm}$, the RMSD of the pressure as less than $1 \mathrm{bar}$, and the average speed of the motor when entering steady-state conditions as less than $50 \mathrm{rpm}$ (Figure 5 and Table 2). 


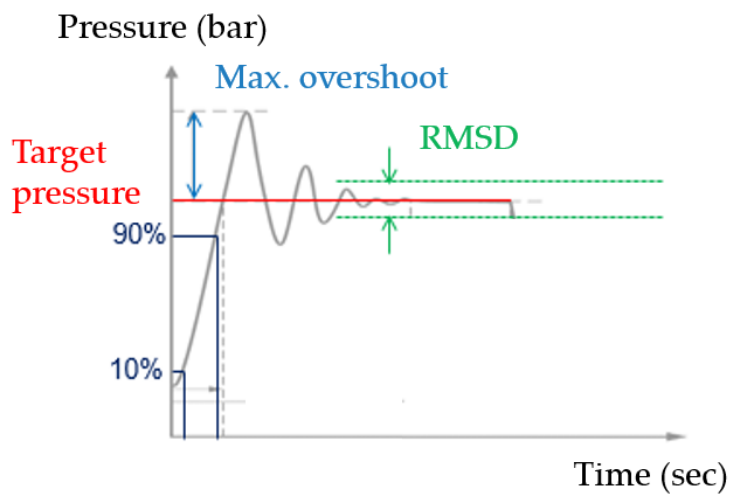

Figure 5. Illustration of the experimental performance indicators.

Table 2. Target values of the performance indicators.

\begin{tabular}{ll}
\hline Performance Indicators & Targets \\
\hline Pressure at the hydraulic cylinder & Max. overshoot less than $5 \mathrm{bar}$ \\
& RMSD less than $1 \mathrm{bar}$ \\
Cylinder position & Max. overshoot less than $1 \mathrm{~mm}$ \\
& RMSD less than $0.5 \mathrm{~mm}$ \\
Response time & less than $3 \mathrm{~s}$ \\
Average speed of the motor & less than $50 \mathrm{rpm}$ \\
\hline
\end{tabular}

\subsection{Multi-Target Positions, Single Target Speed, and Single Target Pressure Control Experiments}

The multi-target positions, single target speed, and single target pressure control experiments are performed to investigate the performance of the proposed system in terms of its speed control, pressure control and V-P switchover point control.

Parameters in the experiment are defined as:

1. Response time: as the definition in the previous section.

2. Position: the position of the cylinder away from the B side.

3. Pressure_1: the pressure value at the pump outlet.

4. Pressure_2: the pressure value at the hydraulic cylinder.

In order to examine the performance of the proposed system under different position sections, flow velocities and sampling frequencies, experiment sets are list below:

1. Sampling frequency: $500 \mathrm{~Hz}$

When the hydraulic cylinder reached the target V-P switchover position in the velocity-control mode, it was pulled back through a distance of $2 \mathrm{~mm}$, and the system then switched to the pressure-control mode to execute the packing stage. Note that the pull-back distance ( $2 \mathrm{~mm})$ was set as the minimum value possible under a sampling frequency of $500 \mathrm{~Hz}$. The corresponding experimental targets are shown in Table 3 for V-P switchover positions (35mm, $50 \mathrm{~mm}$ and $65 \mathrm{~mm})$.

Table 3. Experiment sets under a sampling frequency of $500 \mathrm{~Hz}$.

\begin{tabular}{ccc}
\hline Target Position $(\mathbf{m m})$ & Target Velocity $(\mathbf{m m} / \mathbf{s})$ & Target Pressure (bar) \\
\hline $35 \rightarrow 33$ & $50-80$ & 100 \\
$50 \rightarrow 48$ & $50-80$ & 110 \\
$65 \rightarrow 63$ & $50-80$ & 120 \\
\hline
\end{tabular}


2. Sampling frequency: $1000 \mathrm{~Hz}$

The experimental procedure was the same as that described above and shown in Table 4, with the exception that the pull-back distance was set as $1 \mathrm{~mm}$ (i.e., the minimum attainable distance under a sampling frequency of $1000 \mathrm{~Hz}$ ).

Table 4. Experiment sets under a sampling frequency of $1000 \mathrm{~Hz}$.

\begin{tabular}{ccc}
\hline Target Position $(\mathbf{m m})$ & Target Velocity $(\mathbf{m m} / \mathbf{s})$ & Target Pressure (bar) \\
\hline $35 \rightarrow 34$ & $50-80$ & 100 \\
$50 \rightarrow 49$ & $50-80$ & 110 \\
$65 \rightarrow 64$ & $50-80$ & 120 \\
\hline
\end{tabular}

Note that the entry $50 \mathrm{~mm} \rightarrow 48 \mathrm{~mm}-80 \mathrm{~mm} / \mathrm{s}-110$ bar in the following section indicates that the cylinder is displaced through a distance of $50 \mathrm{~mm}$ at a speed of $80 \mathrm{~mm} / \mathrm{s}$ and is then pulled back through a distance of $2 \mathrm{~mm}$ to $48 \mathrm{~mm}$. The system then switches to the packing stage with a constant packing pressure of 110 bar.

\section{Results}

\subsection{Results of Multi-Target Positions, Single Target Speed, and Single Target Pressure Control Experiments}

The results demonstrate that the behavior of the injection molding process is successfully simulated, as the case $65 \mathrm{~mm} \rightarrow 63 \mathrm{~mm}-80 \mathrm{~mm} / \mathrm{s}-120 \mathrm{bar}, 500 \mathrm{~Hz}$ (Figure 6) shown below. In addition, it is important to look into the following typical phenomenon in the results:

A severe pressure drop at pressure 1 can be observed around the time at $1.75 \mathrm{~s}$, which is the point of the V-P transition (Figure $6 \mathrm{~b}, \mathrm{c}$ ). A slope change of pressure 1 and pressure 2 from $0.5 \mathrm{~s}$ to $1.75 \mathrm{~s}$ shows the effect of the spring resistance. Caused by the pull-back motion, pressure 1 occurs a pressure drop after the V-P transition, note that it indicates the pressure at the outlet of the pump which is not a concern. However, pressure 2 performs steadily even during the pull-back motion, which meets the expected results (Figure 6d).

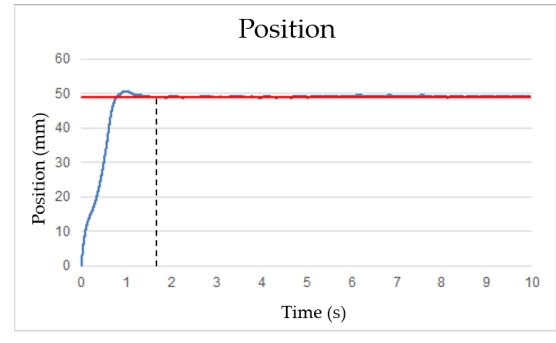

(a) position.

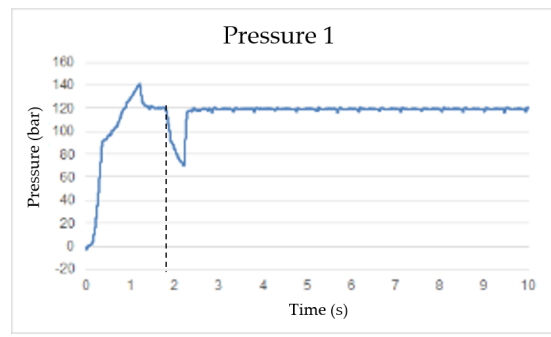

(c) pressure 1

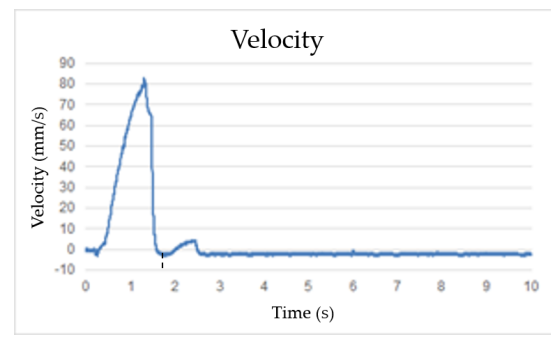

(b) velocity.

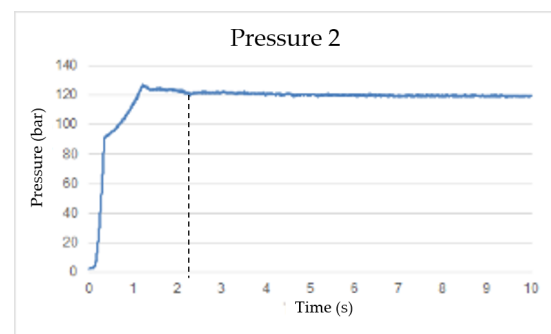

(d) pressure 2 .

Figure 6. Experiment results for the case $65 \mathrm{~mm} \rightarrow 63 \mathrm{~mm}-80 \mathrm{~mm} / \mathrm{s}-120$ bar with, (a) position, (b) velocity, (c) pressure 1 and (d) pressure 2. 
Figure 7 shows the velocity control results under different experiment sets. It can be seen that the flow velocity holds around $50 \mathrm{~mm} / \mathrm{s}$ for about $0.3 \mathrm{~s}$, however, the velocity in Figure $7 \mathrm{~b}, \mathrm{c}$ (Target velocity higher than $50 \mathrm{~mm} / \mathrm{s}$ ) case dropped almost immediately once it reached the target flow velocity. Additionally, the flow velocity rose up after the V-P transition in order to compensate for the effect on pressure due to the pull-pack motion, and then enter the steady-state. A similar effect occurs at the results of the motor speed (Figure 8), the speed reversed after the V-P transition, then leaped up to hold the target pressure.

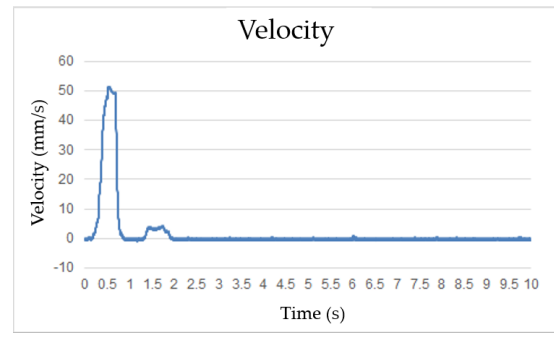

(a) Velocity result of $35 \mathrm{~mm} \rightarrow 33$ $\mathrm{mm}-50 \mathrm{~mm} / \mathrm{s}-100$ bar.

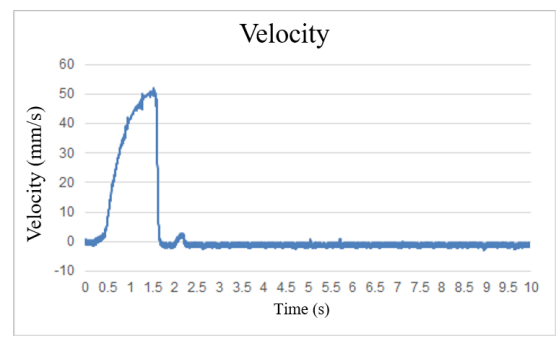

(b) Velocity result of $65 \mathrm{~mm} \rightarrow 64$ $\mathrm{mm}-50 \mathrm{~mm} / \mathrm{s}-120$ bar.

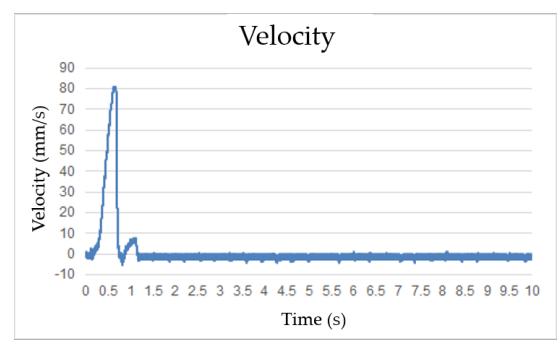

(c) Velocity result of $35 \mathrm{~mm} \rightarrow 34$ $\mathrm{mm}-80 \mathrm{~mm} / \mathrm{s}-100$ bar.

Figure 7. Comparison of velocity control results under different conditions.

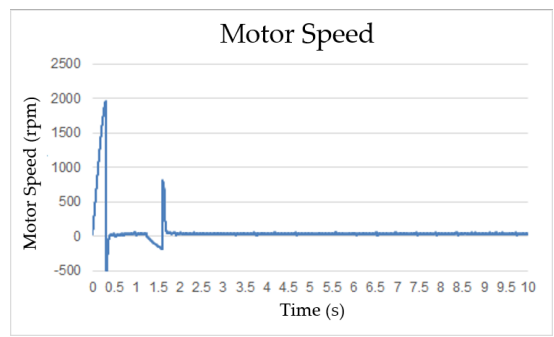

(a) Motor speed result of $35 \mathrm{~mm} \rightarrow 33$ $\mathrm{mm}-80 \mathrm{~mm} / \mathrm{s}-100$ bar.

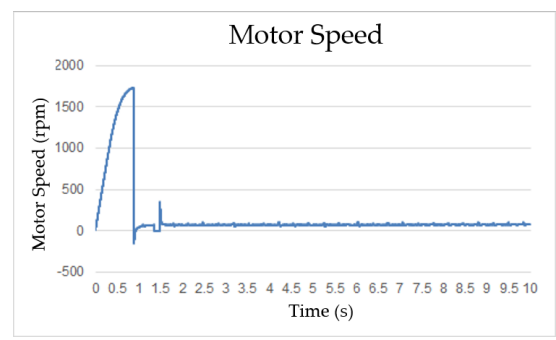

(b) Motor speed of $65 \mathrm{~mm} \rightarrow 64 \mathrm{~mm}-80$ $\mathrm{mm} / \mathrm{s}-120$ bar.

Figure 8. Motor speed results under different conditions.

Figure 9 demonstrates the results of the multi-positions control, the red lines stand for the second target position. The slope indicates the moving forward speed of the hydraulic cylinder, which is positively correlated with the target velocity (Figure 9a,b). The maximum overshoot of the position occurs right before the V-P transition, then the position holds at the second target position steadily.

Comparison of the results are list below in Tables 5-10, with the value out of standards colored. The results of response time performed well under all the circumstance, moreover, the response time decreases as the velocity increases. RMSD of the position sometimes exceeded the preset value under $500 \mathrm{~Hz}$, on the contrary, the results were qualified under $1000 \mathrm{~Hz}$. The pressure at the cylinder (pressure 2) was challenged to meet the target with $500 \mathrm{~Hz}$ under all the experiment sets. In contrast, overshoot and RMSD were successfully controlled at the sampling frequency of $1000 \mathrm{~Hz}$. 


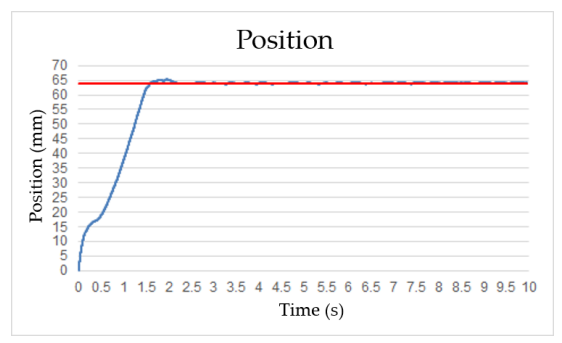

(a) Position result of $65 \mathrm{~mm} \rightarrow 64$ $\mathrm{mm}-50 \mathrm{~mm} / \mathrm{s}-120$ bar.

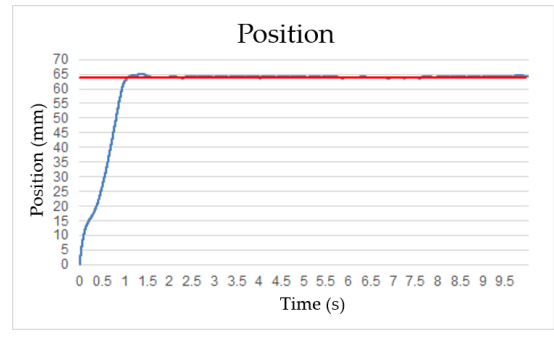

(b) Velocity result of $65 \mathrm{~mm} \rightarrow 64$ $\mathrm{mm}-80 \mathrm{~mm} / \mathrm{s}-120$ bar.

Figure 9. Comparison of position control results under different flow velocities.

Table 5. The $35 \mathrm{~mm} \rightarrow 33 \mathrm{~mm}-100$ bar, $500 \mathrm{~Hz}$.

\begin{tabular}{|c|c|c|c|c|c|c|c|c|}
\hline \multirow{2}{*}{ Velocity $(\mathrm{mm} / \mathrm{s})$} & \multirow{2}{*}{ Response Time (s) } & \multicolumn{2}{|c|}{ Position (mm) } & \multicolumn{2}{|c|}{ Pressure 1 (bar) } & \multicolumn{2}{|c|}{ Pressure 2 (bar) } & \multirow{2}{*}{ Motor Speed (rpm) } \\
\hline & & Overshoot & RMSD & Overshoot & RMSD & Overshoot & RMSD & \\
\hline 50 & 1.64 & 0.17 & 0.1 & 28.29 & 0.85 & 4.82 & 0.55 & 39.7 \\
\hline 70 & 1.55 & 0.14 & 0.16 & 52.95 & 0.77 & 4.8 & 0.95 & 38.71 \\
\hline 80 & 1.45 & 0.6 & 0.29 & 70.7 & 0.85 & 4.7 & 0.34 & 38.28 \\
\hline
\end{tabular}

Table 6. $35 \mathrm{~mm} \rightarrow 34 \mathrm{~mm}-100$ bar, $1000 \mathrm{~Hz}$

\begin{tabular}{cccccccccc}
\hline \multirow{2}{*}{ Velocity (mm/s) } & \multirow{2}{*}{ Response Time (s) } & \multicolumn{2}{c}{ Position $\mathbf{( m m )}$} & \multicolumn{2}{c}{ Pressure 1 (bar) } & \multicolumn{3}{c}{ Pressure 2 (bar) } \\
\cline { 3 - 7 } & & Overshoot & RMSD & Overshoot & RMSD & Overshoot & RMSD & Motor Speed (rpm) \\
\hline 50 & 1.39 & 0.15 & 0.27 & 5.22 & 0.69 & 4.6 & 0.48 & 45.7 \\
60 & 1.21 & 0.23 & 0.13 & 7.19 & 0.7 & 4.9 & 0.36 & 43.85 \\
70 & 1.18 & 0.43 & 0.14 & 10.04 & 0.95 & 3.79 & 0.81 & 48.71 \\
80 & 1.08 & 0.59 & 0.16 & 14.76 & 0.84 & 3.7 & 0.56 & 45.28 \\
\hline
\end{tabular}

Table 7. $50 \mathrm{~mm} \rightarrow 48 \mathrm{~mm}-110$ bar, $500 \mathrm{~Hz}$

\begin{tabular}{ccccccccc}
\hline \multirow{2}{*}{ Velocity (mm/s) } & \multirow{2}{*}{ Response Time (s) } & \multicolumn{2}{c}{ Position $\mathbf{( m m )}$} & \multicolumn{2}{c}{ Pressure 1 (bar) } & \multicolumn{2}{c}{ Pressure 2 (bar) } & \multirow{2}{*}{ Motor Speed (rpm) } \\
\cline { 3 - 7 } & & Overshoot & RMSD & Overshoot & RMSD & Overshoo & RMSD & \\
\hline 50 & 1.99 & 0.18 & 0.1 & 26.3 & 0.82 & 4.8 & 0.49 & 41.52 \\
60 & 1.89 & 0.5 & 0.4 & 38.26 & 0.78 & 5.9 & 0.77 & 42.69 \\
70 & 1.85 & 0.24 & 0.15 & 50.95 & 0.75 & 5.56 & 0.46 & 42.91 \\
80 & 1.8 & 0.7 & 0.59 & 60.38 & 0.83 & 4.91 & 0.28 & 43.79 \\
\hline
\end{tabular}

Table $8.50 \mathrm{~mm} \rightarrow 49 \mathrm{~mm}-110$ bar, $1000 \mathrm{~Hz}$

\begin{tabular}{ccccccccc}
\hline \multirow{2}{*}{ Velocity (mm/s) } & \multirow{2}{*}{ Response Time (s) } & \multicolumn{2}{c}{ Position $\mathbf{( m m )}$} & \multicolumn{2}{c}{ Pressure 1 (bar) } & \multicolumn{2}{c}{ Pressure 2 (bar) } & \multirow{2}{*}{ Motor Speed (rpm) } \\
\cline { 3 - 8 } & & Overshoot & RMSD & Overshoot & RMSD & Overshoot & RMSD & \\
\hline 50 & 1.6 & 0.31 & 0.11 & 9.79 & 0.65 & 4.8 & 0.3 & 43.52 \\
60 & 1.45 & 0.63 & 0.28 & 12.28 & 0.64 & 4.2 & 0.5 & 42.69 \\
70 & 1.24 & 0.53 & 0.28 & 19.13 & 0.69 & 4.95 & 0.41 & 45.91 \\
80 & 1.19 & 0.5 & 0.26 & 26.4 & 0.61 & 4.8 & 0.6 & 46.79 \\
\hline
\end{tabular}

Table $9.65 \mathrm{~mm} \rightarrow 63 \mathrm{~mm}-120$ bar, $500 \mathrm{~Hz}$

\begin{tabular}{cccccccccc}
\hline \multirow{2}{*}{ Velocity (mm/s) } & \multirow{2}{*}{ Response Time (s) } & \multicolumn{2}{c}{ Position $\mathbf{( m m )}$} & \multicolumn{2}{c}{ Pressure 1 (bar) } & \multicolumn{2}{c}{ Pressure 2 (bar) } \\
\cline { 3 - 7 } & & Overshoot & RMSD & Overshoot & RMSD & Overshoot & RMSD & Motor Speed (rpm) \\
\hline 50 & 3.13 & 0.15 & 0.2 & 9.21 & 0.65 & 6.0 & 0.72 & 47.58 \\
60 & 2.75 & 0.25 & 0.15 & 14.21 & 0.83 & 7.3 & 0.48 & 48.38 \\
70 & 2.65 & 0.3 & 0.54 & 17.7 & 0.75 & 7.35 & 0.49 & 49.05 \\
80 & 2.45 & 0.22 & 0.21 & 21.79 & 0.95 & 6.99 & 0.45 & 49.86 \\
\hline
\end{tabular}


Table $10.65 \mathrm{~mm} \rightarrow 64 \mathrm{~mm}-120$ bar, $1000 \mathrm{~Hz}$

\begin{tabular}{|c|c|c|c|c|c|c|c|c|}
\hline \multirow{2}{*}{ Velocity $(\mathrm{mm} / \mathrm{s})$} & \multirow{2}{*}{ Response Time (s) } & \multicolumn{2}{|c|}{ Position (mm) } & \multicolumn{2}{|c|}{ Pressure 1 (bar) } & \multicolumn{2}{|c|}{ Pressure 2 (bar) } & \multirow{2}{*}{ Motor Speed (rpm) } \\
\hline & & Overshoot & RMSD & Overshoot & RMSD & Overshoot & RMSD & \\
\hline 50 & 2.15 & 0.26 & 0.14 & 7.87 & 0.83 & 4.2 & 0.85 & 46.58 \\
\hline 60 & 1.78 & 0.3 & 0.33 & 11.36 & 0.92 & 4.2 & 0.78 & 46.38 \\
\hline 70 & 1.63 & 0.09 & 0.31 & 15.94 & 0.88 & 4.8 & 0.79 & 47.05 \\
\hline 80 & 1.56 & 0.33 & 0.21 & 20.01 & 0.85 & 4.93 & 0.65 & 47.86 \\
\hline
\end{tabular}

\section{Discussion}

Observing the experimental results presented in Tables 5-10, as the target position increases from $35 \mathrm{~mm}$ to $65 \mathrm{~mm}$, the response time increases but still meet the standard. At the sampling frequency of $500 \mathrm{~Hz}$, pressure at the front of the cylinder sometimes exceeds the preset value. However, all the targets are satisfied under the sampling frequency of $1000 \mathrm{~Hz}$. Moreover, the response time under $500 \mathrm{~Hz}$ is a lot greater under $1000 \mathrm{~Hz}$, especially at high target pressure. The response time is crucial for the pull-back motion since the long response time has a risk of affecting the following process. When applying a higher target velocity, the PID controller requires larger gain parameters $\left(\mathrm{K}_{p}, \mathrm{~K}_{i}\right.$, and $\mathrm{K}_{d}$ ) to achieve expected performances. However, the PID controller occurs integral windup as the limitation of the system, the motor driver will finally end up with servo off which be incapable of performing pressure control.

Due to the analog feedback signal noise of the position transducer, the minimum distance of the pull-back motion is tested and turns out to be $2 \mathrm{~mm}$ under a sampling rate of $500 \mathrm{~Hz}$ and $1 \mathrm{~mm}$ under $1000 \mathrm{~Hz}$. Consequently, the sampling frequency does have an impact on the accuracy of the position control. Also, applying a filter or a proper signal process may improve the precision of the position control. By the way, the pull-back motion is controlled by the open direction of the servo proportional valve. Input a positive voltage to the valve causes the hydraulic cylinder moving backward, since the back pressure in the system, the pull-back speed, and distance depends on the opening size of the servo proportional valve and the PID parameters. The experiment results indicate that the PID parameters have little effect on the backward speed of the cylinder, and the pull-back motion is dominated by the opening size of the servo proportional valve (Figure 4).

Though the results are satisfying, there is space for improvement: Injection molding machines in industrial using can have the injection speed much higher than the target speed $(80 \mathrm{~mm} / \mathrm{s})$ in the present study. Due to the limitation of the hydraulic cylinder stroke (170 $\mathrm{mm}$ in the study), the velocity control stage lasts for too short which the cylinder reaches the target position almost simultaneously when the speed reaches target value (Figure 6b). To solve the problem, it could be necessary to run the experiments with an injection molding machine. However, experiments with an injection molding machine could be more complicated since the temperature of the polymer melt and material used for the experiment needs to be taken into account.

\section{Conclusions}

This study has constructed a servo-hydraulic system to simulate the main processes of an injection molding machine. The experimental results support the following main conclusions.

1. The proposed PID controller successfully detects the designed V-P switchover point and switches from the constant-velocity mode to a constant-pressure mode accordingly with the servo proportional valve. The opening size of the valve should be carefully designed to accomplish the pull-back motion.

2. The response time and the pressure overshoot at the cylinder rise as the target pressure increases. In the study of Chen et al. [14], performances of the PID controller and self-tuning fuzzy PID controller have been compared. By introducing the self-tuning fuzzy PID controller in the system can improve the response time, pressure RMSD and pressure overshoot. 
3. When the sampling frequency is set as $1000 \mathrm{~Hz}$, the system achieves all of the displacement, velocity, and pressure performance targets with the exception of the maximum overshoot of the pump outlet pressure in performing V-P switchover.

4. In practical applications, the optimal pull-back distance of the cylinder prior to the packing stage depends not only on machine performance but also on polymer resin properties, and quality requirements of the product, etc. In general, the position control accuracy of servo-hydraulic systems depends on the sampling rate. For the system developed in the present study, a pull-back distance of $1 \mathrm{~mm}$ can be achieved using a sampling frequency of $1000 \mathrm{~Hz}$.

Author Contributions: conceptualization, S.-J.H.; data curation, C.-Y.L.; formal analysis, C.-Y.L. and F.-C.S.; funding acquisition, S.-J.H.; investigation, C.-Y.L. and F.-C.S.; methodology, K.-T.W.; project administration, K.-T.W., H.-H.L. and S.-J.H.; Software, K.-T.W.; supervision, S.-J.H.; validation, H.-H.L.; writing一original draft, F.-C.S.; writing-review and editing, H.-H.L. These authors contributed equally to this work.

Funding: Funding of this project is from the Metal Industries Research \& Development Centre (MIRDC) and SYNTEC company.

Acknowledgments: The authors would like to thank the Metal Industries Research \& Development Centre (MIRDC) and SYNTEC for funding this project.

Conflicts of Interest: To the best of our knowledge, the named authors have no conflict of interest, financial or otherwise.

\section{References}

1. Chen, P.C. Study on the Response Time for a Super High Speed Servo-Hydraulic System and Components. Master's Thesis, Chung Yuan Christian University, Taoyuan City, Taiwan, 2003.

2. Chiang, M.H.; Chen, C.C.; Kuo, C.F.J. The high response and high efficiency velocity control of a hydraulic injection molding machine using a variable rotational speed electro-hydraulic pump-controlled system. Int. J. Adv. Manuf. Technol. 2009, 43, 841-851. [CrossRef]

3. long, Q. Current State, Problems and the Innovative Solution of Electro-hydraulic Technology of Pump Controlled Cylinde. Chin. J. Mech. Eng. 2008, 44, 87-92.

4. Tseng, C.J. Modulized Real-time Control Technology for Injection Molding Machines. Ph.D. Thesis, National Sun Yat-sen University, Kaohsiung, Taiwan, 2000.

5. Lin, S.L. Injection Molding of Plastic; Publishing House of Mechanical Technology: Taipei, Taiwan, 1990.

6. Tsai, C.C.; Kao, H.E.; Hsieh, S.M.; Lin, S.C. Modeling and Gain-scheduling PI Injection Velocity Control of an Ultra-high-speed Plastic Injection Molding Machine. J. Technol. 2006, 21, 317-328.

7. Cheng, P.C. A Study of High Precision Servo Position Control of Hydraulic System under Low Speed. Master's Thesis, National Cheng Kung University, Tainan, Taiwan, 2003.

8. Lai, J.Y.; Chen, Y.R. Adaptive flow rate control of a hydraulic proportional valve. JSME Int. J. 1992, 35, 582-590. [CrossRef]

9. Yang, Y.; Gao, F. Adaptive control of the filling velocity of thermoplastics injection molding. Control Eng. Pract. 2000, 8, 1285-1296. [CrossRef]

10. Bader, C.; Greco, L. Process for Determining the Changeover Point When Producing Plastic Injection Mouldings and Die-Castings. U.S. Patent 5,665,283, 9 September 1997.

11. Chen, Y.S.; Wu, K.T.; Tsai, M.H.; Hwang, S.J.; Lee, H.H.; Peng, H.S.; Chu, H.Y. Adaptive process control of the changeover point for injection molding process. J. Low Freq. Noise Vib. Active Control 2019. [CrossRef]

12. Schiffers, I.R. Adaptive process control for stabilizing the production process in injection moulding machines. In Proceedings of the 10th International Fluid Power Conference (10. IFK), Dresden, Germany, 8-10 March 2016. 
13. Kamiguchi, M.; Umemoto, H. Suckback Method and Apparatus in an Injection Molding Machine. U.S. Patent 4,812,268, 14 March 1989.

14. Chen, C.J.; Wu, K.T.; Hwang, S.J. Development of a servo-hydraulic system with a self-tuning fuzzy PID controller to simulate injection molding process. In Microsystem Technologies; Springer: Berlin/Heidelberg, Germany, 2018; pp. 1-22. 